\title{
Making hasty decisions
}

When making decisions, we often balance the need to make a choice with reasonable speed against the time required to ensure that the decision is accurate (known as a speed-accuracy trade-off (SAT)). Thura and Cisek now provide evidence for a key role of the basal ganglia in the control of this process.

Although the basal ganglia have been implicated in decision making, their precise contribution has been unclear. Here, the authors addressed this issue using a reach-selection task in macaque monkeys.

In each trial, a monkey observed a set of 15 tokens 'jumping', at a rate of one every $200 \mathrm{~ms}$, into one of two targets. The monkey was required to guess which target would eventually contain the most tokens and indicate that choice by moving a handle towards the target at any point in the task. This enabled the authors to distinguish neural events, related to deliberation (weighing up of sensory evidence and target selection), from those related to the commitment to a particular selection and movement. Once the monkey had reached for a target, all of the remaining tokens jumped rapidly to their final positions, either one every $150 \mathrm{~ms}$ in 'slow' blocks of trials (there were 75-125 trials in each block) or one every $50 \mathrm{~ms}$ in 'fast' blocks. Thus, the monkey had to adjust to the SAT context of a particular block: quick (but potentially less accurate) decisions across slow blocks saved less time overall and therefore presented less of an advantage than quick decisions across fast blocks.

During the task, the authors recorded the activity of individual neurons in the globus pallidus (the key output nucleus of the basal ganglia), premotor cortex and primary motor cortex. They found that the activity of cortical neurons strongly correlated with the degree of sensory evidence that accumulated with successive token jumps and was also sensitive to the 'difficulty' of individual trials: easy trials (in which evidence from only a few token jumps enabled a confident decision) corresponded to higher neuronal activity than trials in which the pattern of jumps was ambiguous or misleading. By contrast, activity in globus pallidus neurons seemed mostly insensitive to the sensory evidence, suggesting that these neurons may not be involved in deliberation in this task.

The authors previously hypothesized that the basal ganglia may provide an 'urgency signal' that adjusts the relative weighting of speed versus accuracy during decision making. In support of this possibility, the activity of subsets of globus pallidus neurons gradually increased ('build-up' cells) or decreased ('decreasing' cells) during deliberation. Furthermore, the activity of many of these cells was modulated by the difference in SAT context between fast and slow blocks: build-up cells were more active during fast blocks, when the advantages of urgency were greater, whereas decreasing cells were less active during fast blocks.

The authors argue that output from the basal ganglia, which is modulated by the SAT context, feeds back into the cortical deliberation process, and controls the SAT weighting by determining the amount of sensory information required for commitment to a particular choice. They also note that the basal ganglia may perform additional roles in other types of decision making, such as value-based or reinforcement-learning tasks.

Katherine Whalley

ORIGINAL ARTICLE Thura, D. \& Cisek, P. The basal ganglia do not select reach targets but control the urgency of commitment. Neuron 30, 1160-1170 (2017)

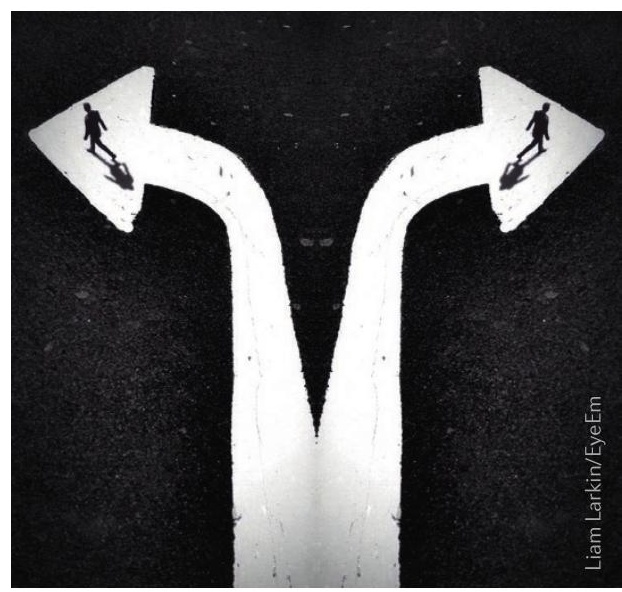

